\title{
OMSD - An open membrane system design tool
}

\section{Aschmoneit, Fynn Jerome; Hélix-Nielsen, Claus}

\section{Published in:}

Separation and Purification Technology

Link to article, DOI:

10.1016/j.seppur.2019.115975

Publication date:

2020

Document Version

Peer reviewed version

Link back to DTU Orbit

Citation (APA):

Aschmoneit, F. J., \& Hélix-Nielsen, C. (2020). OMSD - An open membrane system design tool. Separation and Purification Technology, 233, [115975]. https://doi.org/10.1016/j.seppur.2019.115975

\section{General rights}

Copyright and moral rights for the publications made accessible in the public portal are retained by the authors and/or other copyright owners and it is a condition of accessing publications that users recognise and abide by the legal requirements associated with these rights.

- Users may download and print one copy of any publication from the public portal for the purpose of private study or research.

- You may not further distribute the material or use it for any profit-making activity or commercial gain

- You may freely distribute the URL identifying the publication in the public portal

If you believe that this document breaches copyright please contact us providing details, and we will remove access to the work immediately and investigate your claim 


\title{
OMSD - An Open Membrane System Design Tool
}

\author{
F. J. Aschmoneit, C. Hélix-Nielsen*
}

Department of Environmental Engineering, Technical University of Denmark, Bygningstorvet, 2800 Kgs. Lyngby, Denmark

\begin{abstract}
A tool for design and performance analyses of filtration systems is presented. The tool evaluates the filtration rate and its associated power consumption of complex filtration systems, based on the system design, feed water characteristics, membrane characteristics and operating conditions. It is based on a modular concept, through which system elements, such as filtration modules or pumps, are assembled to build a virtual model of a filtration system. The system performance is based on its operating expenses, evaluated from the filtration rate, the filtrate solute concentration and the power consumption of the pumps and pre-treatment. The system analysis tool is applied to two case studies on the desalination of brackish water and brine. These studies compare various system configurations and operating conditions, based on their energy-efficiency. Based on the findings, general design principles are derived for brackish water and brine desalination. The tool presented here poses the first development stage in the development of an open, versatile and intuitive system design tool. Its open implementation allows for subsequent development contributions of the scientific community. The advantages over existing system tools are its transparency, its free extendibility, and the freedom to implement arbitrary filtration modules, without restrictions to commercial products.
\end{abstract}
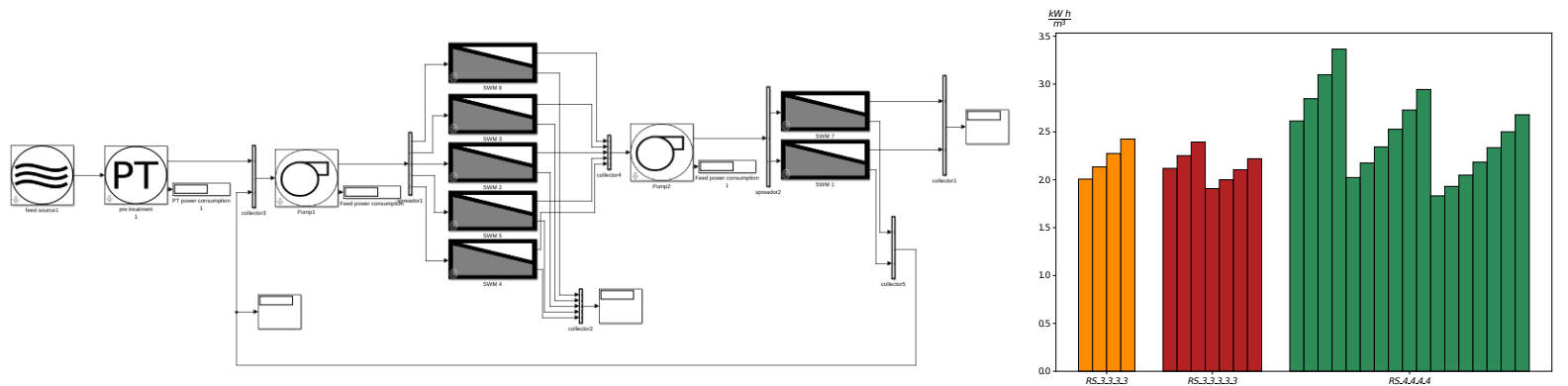

Figure 1: graphical abstract

Keywords: filtration system design, membrane cascade, process optimization, separation efficiency, computer aided engineering

\footnotetext{
* Corresponding author

Email address: clhe@env.dtu.dk (C. Hélix-Nielsen)
} 


\section{Nomenclature}

$\beta \quad$ Spacer voidage

$\Delta p \quad$ hydraulic pressure difference across membrane

$\delta p \quad$ Pressure drop in feed channel

${ }_{5} \quad \dot{m}_{s} \quad$ Solute permeation rate

$\epsilon \quad$ Specific energy consumption

$\eta \quad$ Efficiency of pump

$\nu \quad$ Kinematic viscosity

$\Omega_{P T} \quad$ Membrane area of pre-treatment process

${ }_{10} \quad \pi_{f} \quad$ Feed osmotic pressure

$\rho \quad$ Fluid density

$\tau_{w} \quad$ Wall shear stress

$\theta \quad$ Spacer strand opening angle

A Water permeation coefficient

$A_{P T} \quad$ Pre-Treatment water permeation coefficient

$[m /(s P a)]$

c Solute concentration

$\left[\mathrm{kg} / \mathrm{m}^{3}\right]$

$D \quad$ Solute diffusion coefficient

$d_{h} \quad$ Hydraulic diameter

$f \quad$ Friction number

${ }_{20} \quad h_{c h} \quad$ Feed channel height

$h_{s} \quad$ Module sheet thickness

$J_{w} \quad$ Permeation flux

$k_{m} \quad$ Mass transfer coefficient

$k_{d c} \quad$ Mass transfer correction factor

${ }_{25} \quad l_{m} \quad$ Length of membrane sheet

$l_{f l} \quad$ Filament spacing of feed spacer

$n_{s} \quad$ Number of module sheets 


\begin{tabular}{|c|c|c|}
\hline$P$ & Power consumption & {$[W]$} \\
\hline$p$ & Hydraulic pressure & {$[P a]$} \\
\hline$q_{f}$ & Feed flow rate at module inlet & {$\left[\mathrm{m}^{3} / \mathrm{s}\right]$} \\
\hline$R_{s}$ & Solute rejection coefficient & {$[-]$} \\
\hline$r_{f l}$ & Filament radius of feed spacer & {$[m]$} \\
\hline$r_{p p}$ & Permeate pipe radius of SWM & {$[m]$} \\
\hline$r_{\text {swm }}$ & Outer radius of SWM & {$[m]$} \\
\hline$R e$ & Reynolds number & {$[-]$} \\
\hline$S h$ & Sherwood number & {$[-]$} \\
\hline$U$ & Cross flow velocity in feed channel & {$[\mathrm{m} / \mathrm{s}]$} \\
\hline$w_{m}$ & Width of membrane sheet & {$[m]$} \\
\hline
\end{tabular}

\section{Introduction}

The design of filtration systems is a highly complex task, involving considerations of the solute composition of feed and product waters, their flow rates, and the operating and investment costs. Due to its complexity, the configuration design is typically optimized with the help of computer algorithms. This virtual prototyping allows for cost-efficient system performance analyses, used by membrane suppliers to estimate investment and operating costs of a given filtration problem. BRAKRO[1] was an early system design tool, which solely relied on a manual terminal interface. Through the definition of system requirements in terms of recovery, operating pressure, module type and product quality, BRAKRO would suggest a configuration, including its retentate and permeate solute compositions, the filtration flow rates and the related energy consumption [2]. Based on the same principles, membrane developers have developed their own system design programs. Dow developed ROSA for reverse osmosis systems, which was extended under the name WAVE to also incorporate related membrane processes [3]. Also Toray and SUEZ developed similar design tools for system configurations [4, [5]. These tools incorporate intuitive user-interfaces and tutorial cases. But they also limit their functionality to their own products, which makes cross analyses of configurations with modules from different companies impossible. tools, without being constrained to specific membranes. By contrast, greatest flexibility would be given to the user, by permitting the design of fully customizable module types, and providing full transparency of the applied mathematical models and their underlying operation assumptions. An intuitive user interface would support the simple assembly of configurations and provide sophisticated post-processing options 
for the performance evaluation.

The OMSD tool presented here is a development basis for the above-outlined versatile design tool. At this development stage, the design tool efficiently predicts water filtration through different stages with arbitrary module configurations and recirculation cycles. Among other configurations, this allows for standard tapered-cascade or squared-off designs. The water stream can be measured at any position in the system, allowing for in-depth performance analyses. The tool is implemented in the graphical programming environment Simulink and the underlying computations are executed in Matlab2018b. This software tool combination provides an intuitive user interface for the system configuration, while the complex algorithms are swiftly executed in the background, hidden from the application surface. The $O M S D$ tool can be downloaded from the Git repository [6]. Its open development and free distribution lays the basis for promoting an interest in extending the tool with more functionalities.

This article outlines $O M S D$ 's operation principles with a derivation of the mathematical model for a spiral-wound module (SWM) element. The tool is demonstrated in two model case studies on brackish water and brine desalination, respectively. For both model cases, several system designs are assessed for operating expenses (OPEX), under various operating conditions, and the associated capital expenditures (CAPEX) are discussed.

\section{Methodology}

The filtration module configuration defines the system attributes. System configurations may be arbitrarily complex but they can be reduced to three principal configurations, see Fig. 2. In single-pass $(S P)$ configurations, the modules are aligned in parallel. This simple configuration is favored when the permeate is of acceptable quality after only one filtration stage. Staged configurations use the retentate or permeate of one stage as the feed of the following stage. Retentate-staging $(R S)$ configurations pass the retentate to the subsequent stage. This increases the water recovery significantly, while permeate quality declines with every stage, due to increasing concentrations in the feed stream. If all stages have a similar number of modules, e.g. $R S_{-} 1_{-} 1$ or $R S_{-4} 4-4-4$, the retentate-staging is similar to a single-pass configuration with stacked modules (squared-off configuration). Permeate-staging ( $P S$ ) configurations use one stage's permeate as the feed for the next stage. This refines the permeate, at the cost of a poor recovery. However, through recirculation of the second stage's retentate the recovery can be enhanced. 

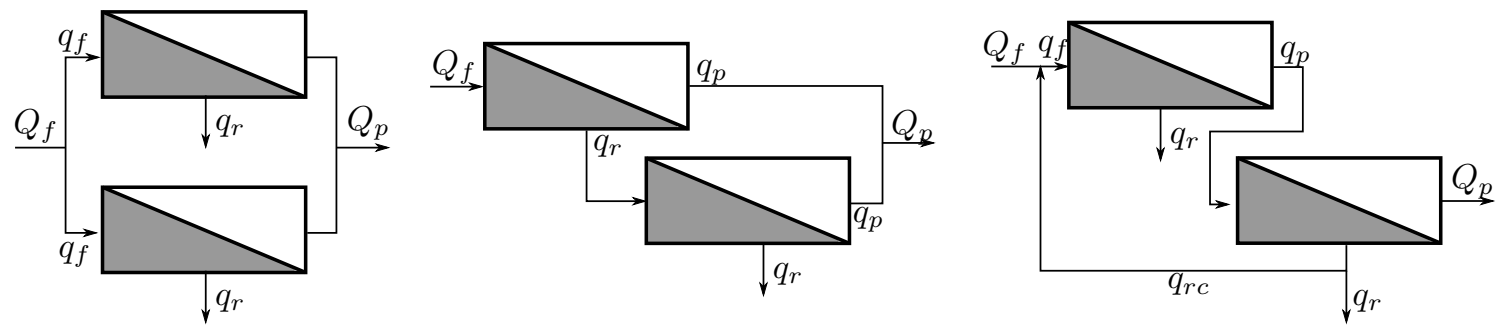

Figure 2: Fundamental module configurations: single-pass SP_2 (left), retentate-staging $R S_{-} 1 \_1$ (middle), permeate-staging with recirculation $P S_{-} 1_{-} 1$ (right). $Q$ denotes a system flow rate and $q$ denotes a module flow rate. The subscripts $f, r, p$ and $r c$ stand for feed, retentate, permeate and recirculations, respectively.

The presented model predicts the evolution of a hydrodynamic state through the filtration system. A hydrodynamic state is defined through the local flow rate $q$, at pressure $p$, with a concentration of $c$. In the following, the local hydrodynamic state is simply referred to by 'stream'. The system analysis tool consists of different elements, which can be multiplied and configured to develop the desired filtration system. Fig. 3 shows the implementation of a $R S_{-} 1 \_1$ system, including a Feed Source element (A), a Pre-Treatment element (B), one Hydraulic Pump (C), six Stream Probes (D) and two RO SWMs (E).

(D1)

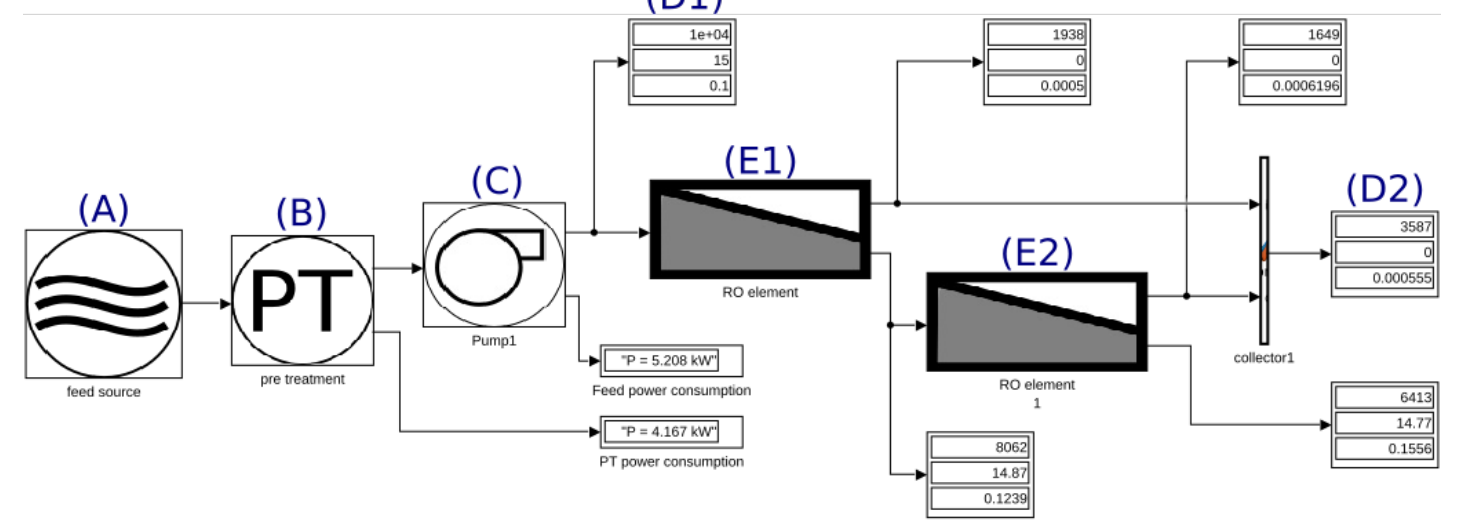

Figure 3: Filtration system in RS_1_1 configuration: (A) Feed source, (B) Pre-Treatment (C) Hydraulic Pump, (D) Stream Probing, (E) ROSWM

(A) The Feed Source is the entry point of the filtration system. Here, the feed flow rate $Q_{f}$ and its solute concentration $c_{f}$ are defined.

(B) The Pre-Treatment element is a simplified model for the calculating the pre-treatment energy demand only. Based on the feed flow rate $Q_{f}$, the utilized membrane area $\Omega_{P T}$, the permeation coefficient $A_{P T}$ and the pump efficiency $\eta$, the power consumption is calculated through:

$$
P_{P T}=\frac{Q_{f}^{2}}{A_{P T} \Omega_{P T} \eta}
$$

(C) The Hydraulic Pump simply pressurizes to stream to the system feed pressure $p_{f}$. Its power consumption is calculated as:

$$
P_{f}=\frac{Q_{f} p_{f}}{\eta}
$$


(D) The Stream Probe is passive, i.e. it doesn't change the stream. It simply measures the stream variables $q([q]=l / h), p([p]=$ bar $)$ and $c([c]=M)$ at the respective position.

(E) The ROSWM module is the most complex system element. It receives the incoming feed stream and derives the outgoing permeate and retentate streams from it. The filtration rate is depending on the membrane characteristics and the SWM module parameters. The underlying algorithm for the SWM model is explained in the following section.

Except for the Stream Probe, all elements' pamareters are configured through a dialog window. The system's filtration performance is evaluated through comparison of the concluding permeate stream (D2) and the primary feed stream (D1). Intermediate probing elements are used for the isolated evaluation of individual stages.

\section{ROSWM Element.}

The most important and most complex element is the RO filtration element. This element is fully generic for all SWM geometries, i.e. module length, radius, number of filtration sheets, embedded spacer characteristics, as well as membrane characteristics can be altered. Once the geometry and membrane characteristics are defined, the element produces permeate and retentate streams, based on one incoming feed stream, see Fig. 3. The permeate and retentate streams are calculated through a feed stream model, which is based on the hydrodynamics in the feed channel. The model executes the following five steps:

1. Feed Cross Flow. The dimensionless cross flow is given by the Reynolds number, on which the subsequent steps depend. It is defined through the mean cross flow velocity $U$, the hydraulic parameter of the feed channel $d_{h}$ and the fluid's kinematic viscosity $\nu$ :

$$
R e=\frac{U d_{h}}{\nu}
$$

The mean cross flow velocity in the feed channel is given by the feed flow rate $q_{f}$ and the cross sectional area of the feed channel. The channel cross section is a thickened spiral curve, which area is similar to the area of a rectangle with the dimensions of the channel height $h_{c h}$ and the membrane width $w_{m}$.

$$
U=\frac{q_{f}}{h_{c h} w_{m}}
$$

The membrane width is a function of the permeate pipe's radius $r_{p p}$, the spiral-wound module's outer radius $r_{s w m}$, its number of membrane sheets, $n_{s}$, and its sheets' thicknesses $h_{s}$. The integral along the spiral path from $r_{p p}$ to $r_{s w m}$ yields the membrane width. Note that the integral must be solved numerically.

$$
w_{m}=\int_{r_{p p}}^{r_{s w m}} \sqrt{\left(\frac{2 \pi}{n_{s} h_{s}} r\right)^{2}+1} d r
$$

Finally, only the hydraulic diameter $d_{h}$ must be defined for the calculation of the Reynolds number (3). The hydraulic diameter is given by four times the cross sectional area, divided by its wetted perimeter. For perfectly cylindrical spacer strands of radius $r_{f l}$ and spacing $l_{f l}$ the hydraulic diameter is:

$$
d_{h}=2 \frac{l_{f l} h_{c h}-\pi r_{f l}^{2}}{l_{f l}+r_{f l}}
$$


2. Feed Pressure Drop. The friction number is the ratio of wall shear stresses and the fluid's kinetic energy. Through the Darcy-Weissbach equation the friction number in ducts can be expressed as [7]:

$$
f=\frac{8 \tau_{W}}{\rho U^{2}}=\frac{2 d_{h}}{\rho U^{2}} \partial_{x} p
$$

The right hand side of the above equation is derived from the wall shear stress definition in terms of the pressure drop $\partial_{x} p$, which is valid for laminar flows only. Investigations on the friction number in feed channels [8] show that the friction number scales with the Reynolds number as:

$$
f=6.23 R e^{-0.3}
$$

This allows the calculation of the pressure drop along the feed channel through the Reynolds number, the channel length $l_{m}$ and the cross flow velocity $U$ :

$$
\delta p=\partial_{x} p l_{m}=R e^{-0.3} \frac{6.23}{2} \frac{l_{m} \rho U^{2}}{d_{h}}
$$

3. Mass Transport. The mass transfer coefficient $k_{m}$ is a measure for how well the solute is mixed in the feed channel, and therefore, to what extend external concentration polarization limits the filtration rate. The Sherwood number $S h$ is a measure for the transport of solute in normal direction of the membrane. It is defined through the rate of change of the solute concentration in the normal direction of the membrane, $\left.\partial_{n} c\right|_{0}$, and the solute concentration difference on the membrane surface and the bulk flow, $\Delta c$.

$$
S h=\frac{\left.d_{h} \partial_{n} c\right|_{0}}{\Delta c}=\frac{d_{h} k_{m}}{D}
$$

where $k_{m}=\left.D \partial_{n} c\right|_{0} / \Delta c$ is the mass transfer coefficient and $D$ is the solute diffusion coefficient.

The mass transfer in empty channels was first defined by Gröber [9], as a scaling law of the flow magnitude, in terms of the Reynolds number $R e$, the ratio of momentum to solute diffusivity, i.e. the Schmidt number $S c$, and the geometry of the channel. Da Costa et al. [10] developed a mass transfer scaling law for spacer-filled channels based on Gröbers theory by including a correction factor $k_{d c}$ :

$$
S h=0.664 k_{d c} R e^{0.5} S c^{0.33}\left(\frac{2 d_{h}}{l_{f l}}\right)^{0.5}
$$

The correction factor accounts for the spacer strand thickness, the strand opening angle $\theta$ and the voidage $\epsilon$ :

$$
k_{d c}=1.654\left(\frac{2 r_{f l}}{h_{c h}}\right)^{-0.039} \epsilon^{0.75}(\sin \theta / 2)^{0.086}
$$

The mass transfer coefficient is calculated from equation 10$]$, with the Sherwood number derived from equations 11 and 12 .

4. Water Permeation Rate. This model assumes a negligible osmotic pressure in the permeate channel. The water flux equation is then expressed in terms of the water permeation coefficient $A$, the feed osmotic pressure $\pi_{f}$, the hydraulic pressure difference across the membrane $\Delta p$ and the afore-derived mass transport coefficient $k_{m}$ :

$$
J_{w}=A\left[\Delta p-\pi_{f} \exp \left(\frac{J_{w}}{k_{m}}\right)\right]
$$


Note that the water flux equation is non-analytic and must be solved numerically. The van't Hoff equation proposes a linear relationship between the osmotic pressure and the solute concentration. Geraldes et al. found this relation to be $\pi_{f}=\left(8.051 \cdot 10^{4} \mathrm{~m}^{2} / \mathrm{s}^{2}\right) c_{f}$. The permeation flux $J_{w}$ allows the evaluation of the total permeation flow rate through the total membrane area $2 n_{s} l_{m} w_{m}$ :

$$
q_{p}=2 n_{s} l_{m} w_{m} J_{w}
$$

5. Solute Permeation Rate. The permeation of solute through the membrane is governed by the solute rejection $R_{s}$, the feed concentration $c_{f}$ and the water permeation $q_{p}$ through:

$$
\dot{m}_{s}=c_{f}\left(1-R_{s}\right) q_{p}
$$

The five evaluation steps above allow the definition of the outgoing stream variables. The permeate stream variables are defined through:

$$
\begin{aligned}
& q_{p}: \text { from equation } 14 \\
& p_{p}=0 \\
& c_{p}=\frac{\dot{m}_{s}}{q_{p}}
\end{aligned}
$$

And the retentate stream variables are:

$$
\begin{aligned}
& q_{r}=q_{f}-q_{p} \\
& p_{r}=p_{p}-\delta p \\
& c_{r}=c_{f}\left(1+R_{s} \frac{q_{p}}{q_{f}}\right)
\end{aligned}
$$

\section{Case Studies}

The system analysis tool is executed on two fictitious desalination problems, in which drinking water is produced from brackish water and brines. The effect of different configurations, feed flow rates and recirculation is assessed for the permeate flow rate, the water recovery, and the power consumption. The only system requirements are a filtration rate of $400 \mathrm{~m}^{3} / \mathrm{d}$ and a permeate $\mathrm{NaCl}$ concentration of at most $1.7 \mathrm{mM}$. For both problems, all configurations utilize the same $S W M$. Its geometry is a 8040 module, with an orthogonal 28 mil feed spacer, and an embedded membrane with $A=5 L M H$ and $R=99.5 \%$. The resultant membrane area equates to $41 \mathrm{~m}^{2}$ per $S W M$. Feed and pre-treatment pumps are operated at $80 \%$ efficiency. The pre-treatment membrane surface area is kept constant at $100 \mathrm{~m}^{2}$ for all cases.

\section{Brackish Water Desalination.}

In the case of brackish water desalination, a feed concentration of $c_{f}=0.2 M$ is assumed. Due to the low concentration and the membranes' high salt rejection, the permeate salt concentration requirement is met after a single stage pass, which allows for retentate staging configurations. The analysis of the brackish 
water desalination therefore concentrates on the optimization of the energy demand, for squared-off configurations, without recirculation.

Since the configuration efficiency depends on the operating conditions, i.e. the feed stream $\left(c_{f}, p_{f}, q_{f}\right)$, an initial analysis on the utilization of the individual stages in a stack of six $S W M s$ is conducted. Fig. 4 shows the recovery per stage of a $R S_{-} 1_{-} 1_{-} 1_{-} 1_{-} 1 \_1$ configuration for various feed flow rates and operating pressures (denoted by $O P 1, \ldots, O P 6)$. The recovery for stage $i$ is defined through:

$$
R^{(i)}=\frac{q_{p}^{(i)}}{q_{f}^{(i)}}
$$

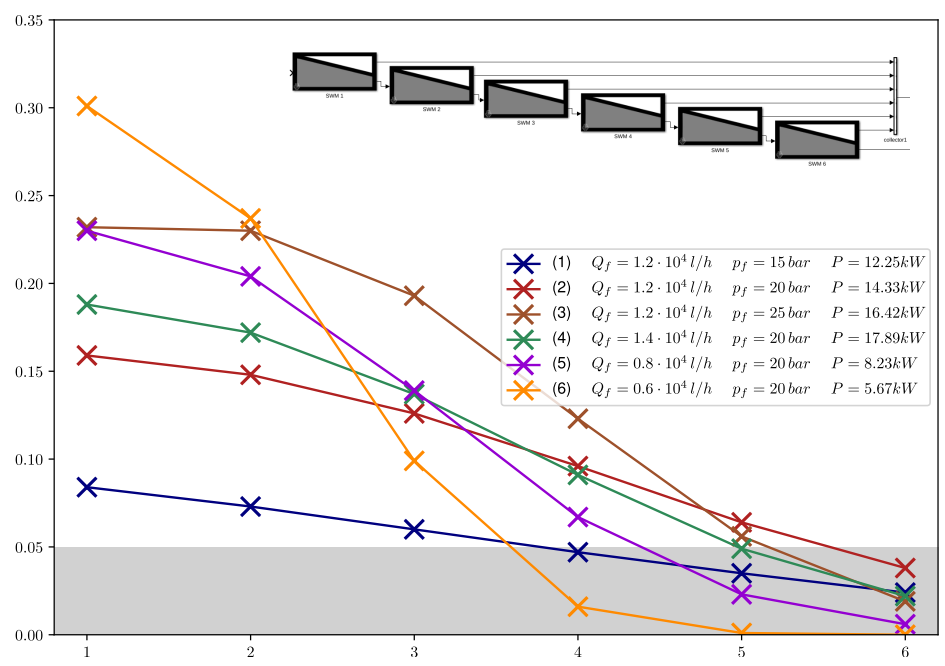

Figure 4: Recovery per stage in $R S_{-} 1 \_1 \_1 \_1 \_1 \_1$ configuration and 5\% recovery limit.

$O P 1$ is operated with a low feed flow rate, which yields a high recovery in the first two stages, but performs poorly in successive stages $\left(R^{(4)}<5 \%\right.$ ), due to a diminishing feed stream. OP6, on the other hand, is operated at low pressure, which is just above the feed osmotic pressure. The successive upconcentration of retentate has a severe effect on the stages' recovery. Also this configuration system shows a recovery of less than $5 \%$ after the third stage. The remaining four operation sets show a wider performance spectrum. Based on these, favorable operating conditions for the subsequent analyses are defined as:

$$
\begin{aligned}
& q_{f} \in(192,336) \frac{m^{3}}{d} \\
& p_{f} \in[18,20,22] \text { bar }
\end{aligned}
$$

The filtration problem is analyzed with the three squared-off configurations $R S_{-}$3_3_3_3, $R S_{-}$3_3_3_3_3

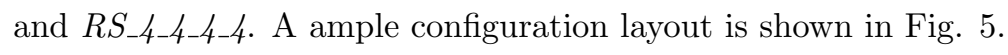




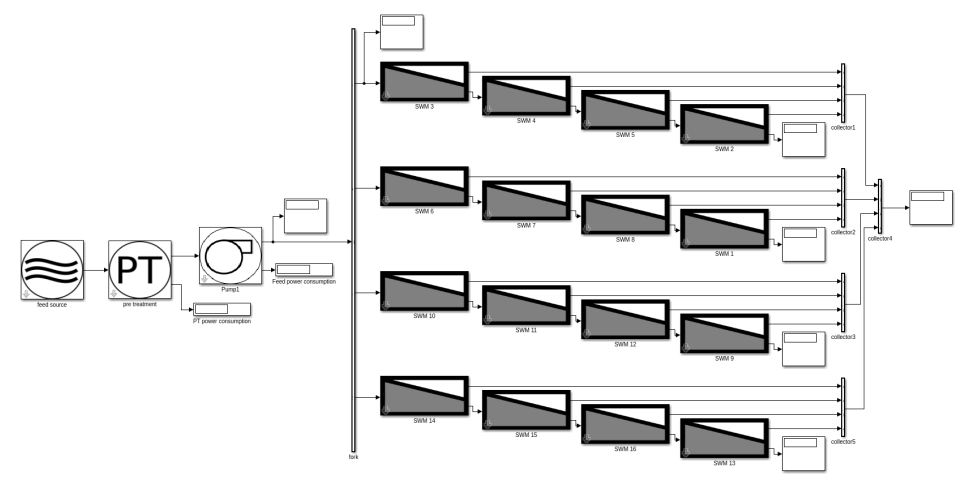

Figure 5: System design export from $O M S D$ : The $R S_{-4-4-4-4}$ configuration for brackish water desalination.

All three configurations are subjected to the operating conditions $(19)$. Fig 6 shows the permeate flow rate of the three configurations, under the applied operating conditions. The graph color indicates the respective configuration and the cross, dot and diamond markers indicate the operating pressure at $18 \mathrm{bar}, 20 \mathrm{bar}$ and $22 \mathrm{bar}$, respectively. The dashed line indicates the permeate flow rate requirement.

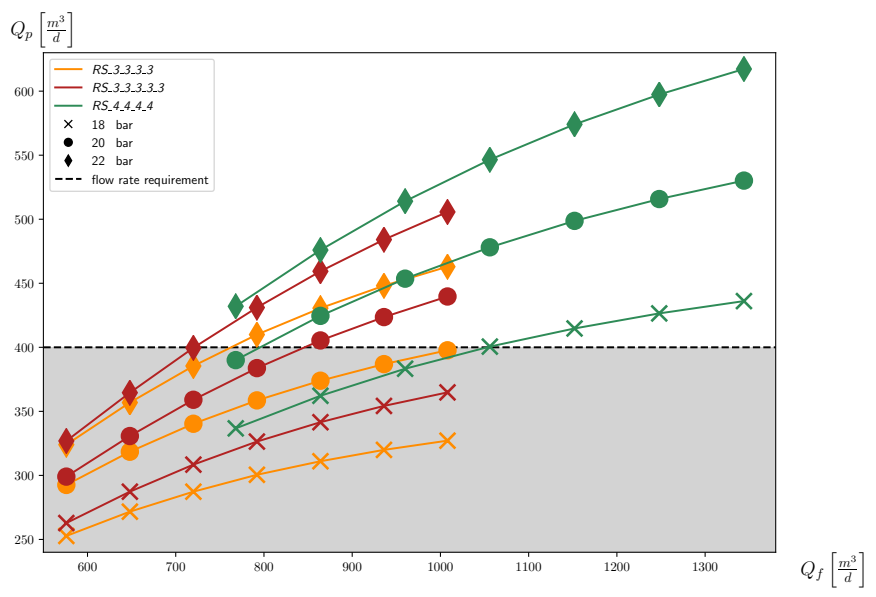

Figure 6: Filtration rates of different configurations, under different operating conditions, for the brackish water desalination case. The dashed line shows the filtration rate requirement.

It is seen that the $2 S_{-}$3_3_3_3 configuration (orange) only meets the permeate flow rate requirement at $22 \mathrm{bar}$ and with a feed flow rate of $\sim 800 \mathrm{~m}^{3} / d$, corresponding to a recovery of $\sim 50 \%$. Further increasing the feed flow rate yields a sublinear increase of the permeate flow rate.

RS_3_3_3_3_3 (red) is arranged through adding a fifth filtration stage to $R S_{-}$3_3_3_3. This configuration satisfies the requirements at $p_{f}=20 \mathrm{bar}$ with $Q_{f} \gtrsim 850 \mathrm{~m}^{3} / d$ or $p_{f}=22 \mathrm{bar}$ and $Q_{f} \gtrsim 750 \mathrm{~m}^{3} / \mathrm{d}$, relating to maximum recoveries of $47 \%$ and $56 \%$, respectively. In agreement with Fig. 4 . RS_3_3_3_3_3 performs slightly better than $R S_{-3} 3_{-3} 3$ 3_3, at the expense of additional SWMs.

$R S$ _4-4-4-4 (green) is arranged through adding a fourth SWM stack to $R S_{-}$3_3_3_3. This configuration can be operated at $p_{f}=18 \mathrm{bar}$ with $Q_{f} \gtrsim 1050 \mathrm{~m}^{3} / d, p_{f}=20 \mathrm{bar}$ with $Q_{f} \gtrsim 800 \mathrm{~m}^{3} / d$ and $p_{f}=22 \mathrm{bar}$ with $Q_{f} \gtrsim 700 \mathrm{~m}^{3} / d$, with maximum recoveries of $38 \%, 50 \%$ and $57 \%$, respectively. This shows how 
additional filtration stacks increase the system performance, at the expense of additional SWMs and an increased feed flow.

As all three designs are operated with one pre-treatment step, one feed pressure pump and no recirculation, the total power consumption is proportional to the operating pressure and greater-than-quadratic with respect to the feed flow rate, see equations (1), (2). At a given operating pressure, configurations $R S \_3 \_3 \_3 \_3$ and $R S \_3 \_3 \_3 \_3 \_3$ therefore have similar a similar power consumption, while $R S \_4-4-4-4$ has a higher power consumption, due to the increased feed flow demand of the fourth SWM stack.

Fig. 7 shows the specific energy demand $\epsilon=P / Q_{p}$, i.e. the energy demand per $1 \mathrm{~m}^{3}$ filtrate, for all operating conditions that passed the filtration requirement of $400 \mathrm{~m}^{3} / d$. Of the investigated configurations and operating conditions, the $R S_{-4-4-4-4}$, operated at $p_{f}=22 \mathrm{bar}$ with $Q_{f}=768 \mathrm{~m}^{3} / d$ yields the lowest energy demand of $\epsilon=1.8 \mathrm{kWh} / \mathrm{m}^{3}$. If operated at $p_{f}=18 \mathrm{bar}$ with $Q_{f}=1343 \mathrm{~m}^{3} / d, R S_{-4}-4-4-4$ operates least efficiently with $\epsilon=3.4 \mathrm{kWh} / \mathrm{m}^{3}$.

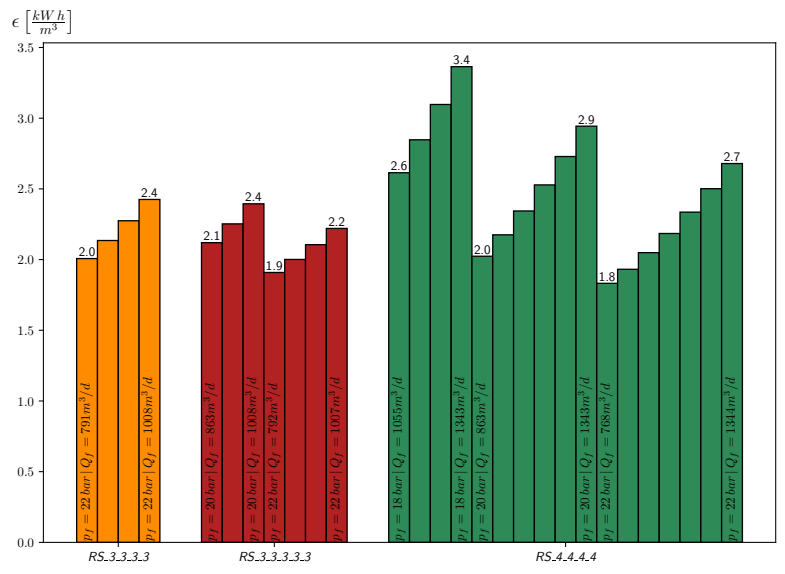

Figure 7: Specific energy for the three configurations and those operating conditions, that meet the filtration rate requirement, for brackish water desalination.

\section{Brine Desalination.}

The brine is assumed to have a $\mathrm{NaCl}$ concentration of $1.5 \mathrm{M}$. For $\mathrm{NaCl}$, this concentration poses the limit of the applicability of van't Hoff's law [11. Due to the high concentration, the drinking water requirement of $c_{p} \leq 1.7 \mathrm{mM}$ cannot be met with only one stage. This allows only for permeate-staging configurations. It was found that two stages filtrate the brine sufficiently. In permeate-staging configurations, it is necessary to pressurize the first stage's permeate, before it enters the second stage as feed. An additional pump is therefore included to drive a two stage permeate-staging system. Due to the reasonably low first stage permeate concentration, the second stage is driven at a significantly lower operating pressure. The second stage retentate has a low $\mathrm{NaCl}$ concentration and is pressurized. It is recirculated and merged with the system feed stream. The recirculation factor $\phi$ defines the fraction of recirculated water in the feed stream. 


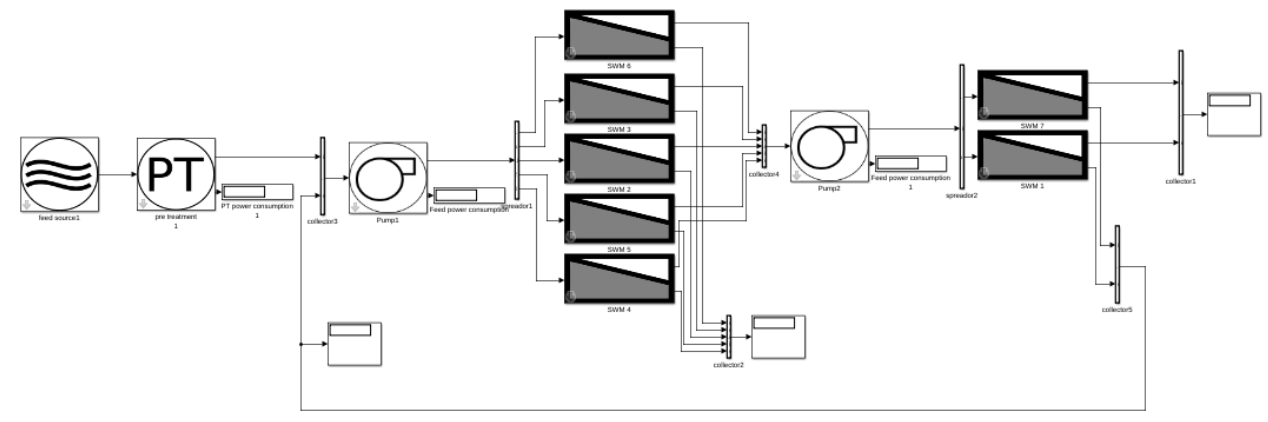

Figure 8: System design export from $O M S D$ : The PS_5_2 configuration for brine desalination.

In order to determine favorable operating conditions a sample PS_5_2 configuration (Fig. 8) is tested for different operating pressures and recirculation fractions. As the first stage recovery is most significant for the system performance, it is driven at only $q_{f}^{(1)}=72 \mathrm{~m}^{3} / d$ per SWM. Its filtration rate is therefore determined by the operating pressure $p_{f}^{(1)}$ and the effective feed concentration, which depends on the recirculation factor $\phi$.

The second stage feed pump controls the filtration rate and the recirculation flow. It is adjusted to yield the desired recirculation flow. Fig. 9 shows the recovery of the first stage as a function of the operating pressure $p_{f}^{(1)}$, for different recirculation ratios $\phi$. As expected, the recovery increases with $p_{f}^{(1)}$, and increasing recirculation ratios $\phi$. A high but reasonable first stage recovery limit was set to $R^{(1)}=80 \%$. This limit is exceeded for high recirculation ratios of $\phi=33 \%$ and $\phi=50 \%$, at operating pressures greater than $p_{f}^{(1)}=77$ bar and $p_{f}^{(1)}=61$ bar, respectively.

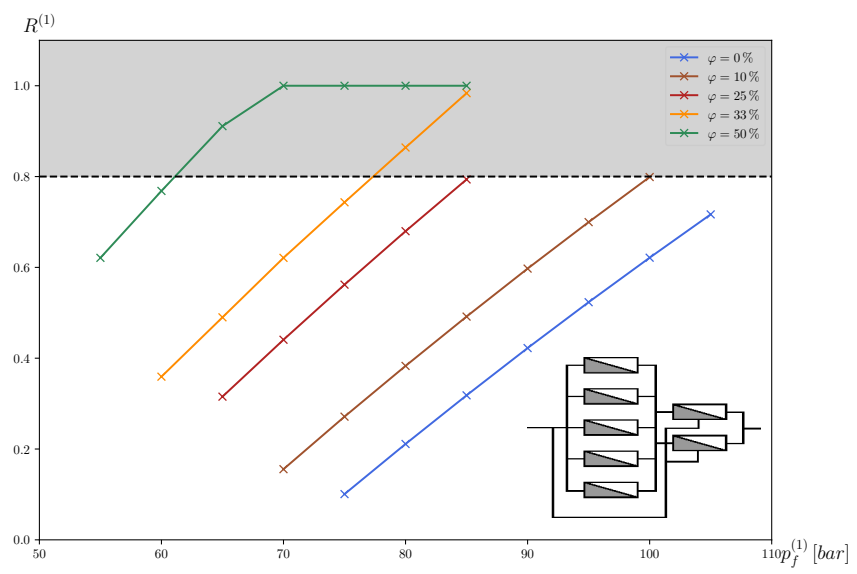

Figure 9: Effect of recirculation and driving pressure on the first stage recovery

Based on this recovery spectrum, solutions to the filtration problem are sought in the range of driving pressures $p_{f}^{(1)} \in[60,90]$ bar. The second stage pump is adjusted to create the specific recirculation flow, which yields the target recovery of $80 \%$ in the first stage. The feed flow rate per SWM is fixed to $q_{f}^{(1)}=72 \mathrm{~m}^{3} / d$ or $q_{f}^{(1)}=108 \mathrm{~m}^{3} / d$. Four characteristic, mutually-dissimilar, two-stage $P S$ configurations 
are applied for the filtration problem: PS_9_6, PS_12_3, PS_14_7, PS_15_5. Fig. 10 shows the total filtration flow rate as a function of the driving pressure $p_{f}^{(1)}$. It is seen that the number of SWMs in the first stage determines the minimum operating pressure, for which the filtration rate requirement of $Q_{p} \geq 400 \mathrm{~m}^{3} / d$ is met: With fewer SWMs in the first stage, a greater operating pressure must be applied. Through increasing the feed flow rate per SWM from $q_{f}=72 \mathrm{~m}^{3} / d$ to $q_{f}=108 \mathrm{~m}^{3} / d$, the filtration rate increase is proportional to the driving pressure. Only the PS_9_6 configuration, operated at low feed flow, does not meet the permeate flow rate requirement for any applied driving pressure.

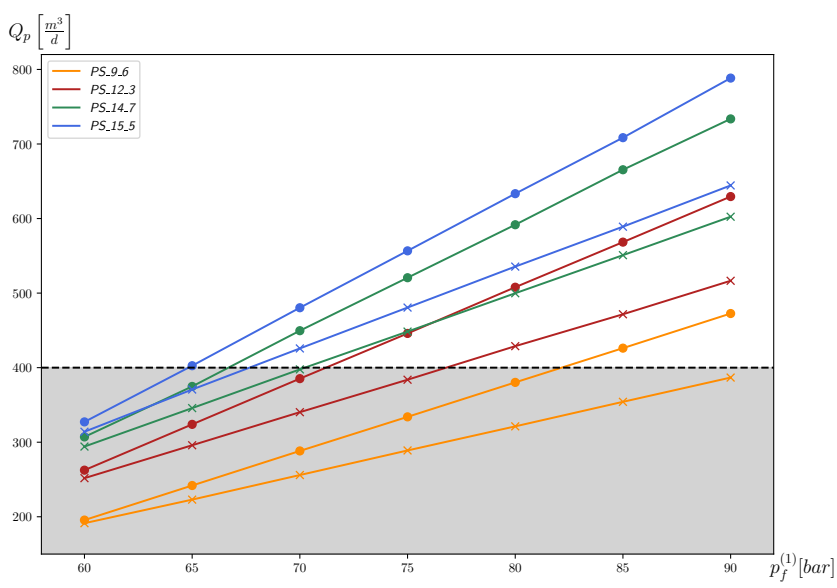

Figure 10: Filtration rates of different configurations, under different operating conditions, for the brine desalination case. The dashed line shows the filtration rate requirement.

The specific energy demand of those configurations and operating conditions, that meet the filtration rate requirement, is plotted in Fig. 11. It is seen that the specific energy demand decreases with higher operating pressures and increases with greater feed flow rates. In the operation mode of constant first stage recovery, the specific energy demand therefore also decreases with a diminishing recirculation. Of the investigated configurations and operating conditions, the $P S_{-} 14_{-} 7$ configuration, operated at $90 \mathrm{bar}$, a total feed flow rate of $Q_{f}=1008 \mathrm{~m}^{3} / d$ and a $\phi=20 \%$ recirculation, is most energy-efficient with $\epsilon=5.9 \mathrm{kWh} / \mathrm{m}^{3}$. 


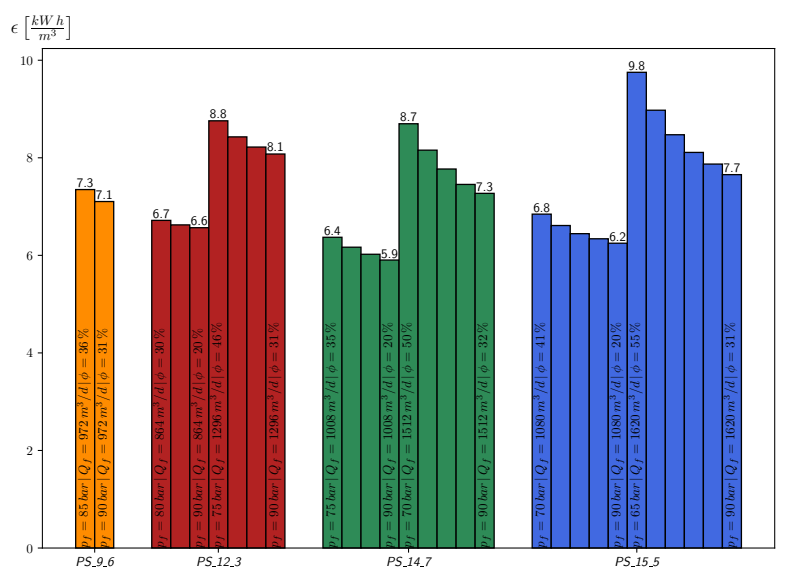

Figure 11: Specific energy for the three configurations and those operating conditions, that meet the filtration rate requirement, for brine desalination.

\section{Discussion.}

The two filtration problems were analyzed through two fundamentally different configuration principles, squared-off retentate-staging and cascade permeate-staging. For different configurations, the operating conditions were optimized to yield a high energy-efficiency, which directly relates to the OPEX. The respective number of filtration elements relates to the CAPEX.

For both configuration design principles, it was found that the OPEX decreases significantly with increasing operating pressures and decreasing feed flow rates. However, there is a practical limit as to how low the feed flow rate can be, before membrane fouling severely reduces the system performance.

For retentate-staging squared-off designs, at constant feed flow, the OPEX is reduced significantly through additional filtration stacks, at the cost of increased CAPEX. However, through adding more stages, the OPEX is reduced insignificantly, while the CAPEX increases.

For permeate-staging cascade configurations, it was found that the recirculation of second stage retentate has a negative effect on the OPEX. Furthermore, through adding more filtration elements to the second stage, the OPEX is reduced insignificantly, while the CAPEX increases.

In general, a filtration system's OPEX is set through the governing filtration design and the applied operating conditions. Its fine-tuning is a trade-off between OPEX and CAPEX.

\section{Conclusion \& Outlook}

A tool for the performance prediction of filtration systems was presented. It was applied to two case studies, in which the energy-efficiency of different configurations was analyzed. These case studies show how the tool can be used in the design of filtration systems. They also indicate, where the design tool shows potential for the next development stage: A model for membrane fouling would allow for transient analyses of the associated performance decline. The implementation of feed quality parameters, which relate to membrane fouling, and which could be adjusted through a more sophisticated pre-treatment 
model, would permit the analyses of minimum feed flow rates and the related performance increase. Following development stages would incorporate compositions of different solutes, temperature-dependent permeation, different module types, heat- and pressure exchangers, and associated membrane processes.

\section{Acknowledgements}

This work was supported by the Innovation Fund Denmark, Innovationsfonden, under the MEMENTO project with grant number 4106-00021B.

\section{References}

[1] R. Quin, Theory and Practise of Reverse Osmosis, Tech. rep. (1985).

[2] B. Parekh (Ed.), Reverse Osmosis Technology - Applications for High-Purity-Water Production, 1st Edition, Marcel Decker Inc, 1988.

[3] $\operatorname{Dow}(\mathrm{Du}$ Pont), ROSA/WAVE

URL https://www.dupont.com/water/design-software.html

[4] SUEZ Water Technologies and Solutions, Winflows

URL https : //www. suezwatertechnologies.com/resources/winflows

[5] Toray, Toray DS

URL https://ap3.toray.co.jp/toraywater/

[6] F. Aschmoneit, OMSD (2019).

URL https://github.com/FynnAschmoneit/OMSD.git

[7] M. H. Chaudhry, Applied Hydraulic Transients, 3rd Edition, Springer New York, 2014.

[8] G. Schock, Mass transfer and pressure loss in spiral wound modules, Desalination 64 (1987) 339-352.

[9] H. Gröber, S. Erk, U. Grigull, Fundamentals of Heat Transfer, 1st Edition, McGraw-Hill Book Company, 1961.

[10] A. R. Da Costa, A. G. Fane, D. E. Wiley, Spacer characterization and pressure drop modelling in spacer-filled channels for ultrafiltration, Journal of Membrane Science 87 (1994) 79-98.

[11] V. Geraldes, V. Semião, M. Norberta De Pinho, Flow and mass transfer modelling of nanofiltration, Journal of Membrane Science 191 (2001) 109-128. 